\title{
Feasibility and Limits of Wi-Fi Imaging
}

\author{
Donny Huang ${ }^{\dagger}$, Rajalakshmi Nandakumar ${ }^{\dagger}$, Shyamnath Gollakota \\ University of Washington \\ \{donnyhuang, rajaln, gshyam\}@cs.washington.edu \\ †Co-primary Student Authors
}

\begin{abstract}
We explore the feasibility of achieving computational imaging using Wi-Fi signals. To achieve this, we leverage multi-path propagation that results in wireless signals bouncing off of objects before arriving at the receiver. These reflections effectively light up the objects, which we use to perform imaging. Our algorithms separate the multi-path reflections from different objects into an image. They can also extract depth information where objects in the same direction, but at different distances to the receiver, can be identified. We implement a prototype wireless receiver using USRP$\mathrm{N} 210 \mathrm{~s}$ at $2.4 \mathrm{GHz}$ and demonstrate that it can image objects such as leather couches and metallic shapes in line-of-sight and non-line-of-sight scenarios. We also demonstrate proofof-concept applications including localization of static humans and objects, without the need for tagging them with RF devices. Our results show that we can localize static human subjects and metallic objects with a median accuracy of 26 and $15 \mathrm{~cm}$ respectively. Finally, we discuss the limits of our Wi-Fi based approach to imaging.
\end{abstract}

\section{Categories and Subject Descriptors Communication \\ Keywords \\ Wireless sensing, Wi-Fi imaging}

C.2.1 [Network Architecture and Design]: Wireless

\section{Introduction}

Is it possible to leverage $\mathrm{Wi}-\mathrm{Fi}$ signals to create images of objects and humans? Given the ubiquity of Wi-Fi signals, a positive answer would allow us to localize static humans even when they do not carry any RF devices, thus enabling pervasive home sensing. It would also enable new applications such as inventory localization - objects such as carts can be tracked without either the need for tagging them with $\mathrm{RF}$ sources or the burden of installation and cost that make

Permission to make digital or hard copies of all or part of this work for personal or classroom use is granted without fee provided that copies are not made or distributed for profit or commercial advantage and that copies bear this notice and the full citation on the first page. To copy otherwise, to republish, to post on servers or to redistribute to lists, requires prior specific permission and/or a fee.

SenSys'14, November 3-6, 2014, Memphis, TN, USA.

Copyright (C) 2014 ACM 978-1-4503-1169-4 ...\$10.00

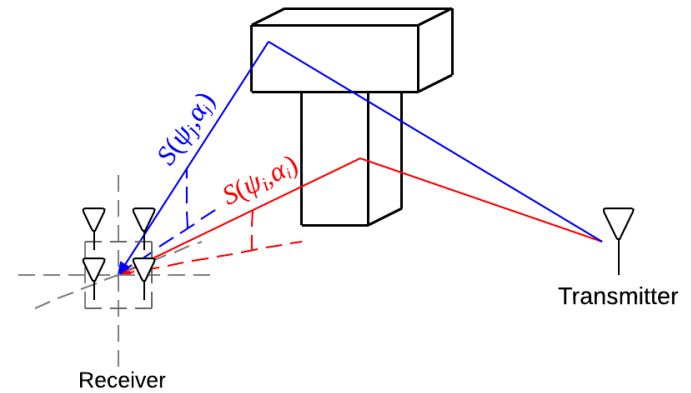

Figure 1-Wision at a high level. Wision uses the transmissions from WiFi devices in the environment to enable imaging on a multi-antenna AP. Our approach leverages multi-path propagation that results in signals bouncing off of objects before they arrive at the AP. These reflections serve the purpose of "lighting" up the objects, which Wision uses for imaging.

vision-based sensing hard to deploy at scale, for example, throughout an entire home or building. Beyond localization, it also creates object detection capabilities for security applications where, using Wi-Fi, one could detect metallic objects hidden in non-line-of-sight scenarios.

While radar systems perform imaging, they are not applicable to Wi-Fi since they require ultra-wideband transmissions with GigaHertz of bandwidth [39, 55]. Wi-Fi, on the other hand, has a relatively narrow bandwidth of $20 \mathrm{MHz}$ that limits the resolution of radar approaches to about 7.5 meters [47, 12]. Further, radar systems use tailor-made transmissions (e.g., FMCW [32]) to perform imaging; in contrast, Wi-Fi uses OFDM modulation that is optimized for communication and not for imaging purposes.

In this paper, we explore the feasibility and limits of performing imaging using Wi-Fi signals. We present Wision that enables computational imaging of objects and humans using Wi-Fi reflections. As shown in Fig. 1 Wision uses the transmissions from Wi-Fi devices in the environment to enable imaging on a multi-antenna AP. Our approach leverages multi-path propagation that results in signals bouncing off of objects before they arrive at the AP. These reflections serve the purpose of "lighting" up the objects, which Wision uses for imaging. The main challenge, however, is that the AP sees a combination of the reflections from multiple objects in the environment - imaging requires an ability to separate these reflected signals at the receiver.

Wision addresses this challenge by leveraging multiple antennas that are increasingly common on Wi-Fi access 


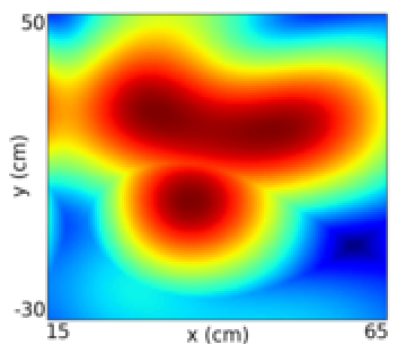

Figure 2-Example image created by Wision. The figure shows the image captured by Wision of a T-shaped metallic object.

points. Wi-Fi APs today come with an ever-increasing number of antennas; 16-32 sectorized antenna Wi-Fi APs are already available on the market [3]. Further, there is a push in the academic community to provide higher network capacity by using large-scale MIMO APs with tens to hundreds of antennas [52, 45, 22].

To understand Wision, consider the wireless signals received by the two-dimensional antenna array shown in the figure. The Wision receiver leverages angle-of-arrival techniques to construct an image that separates the reflections from each azimuthal and elevation angle. Specifically, Wision performs a 2D-FFT operation to separate the multi-path reflections on its antenna array. Intuitively, a signal from an azimuthal and elevation angle results in a specific pattern of phase differences across the receive antennas. Since differences in phase translates into a frequency, each azimuthal and elevation angle corresponds to a specific frequency component at the receiver. Thus, by computing an FFT, the receiver can separate these signals. Wision then constructs an image by measuring the intensities of the multi-path reflections from each azimuthal and elevation angle.

Wision also goes beyond the capabilities of traditional optical cameras, and extracts the depth information. Specifically, since the above FFT operation produces a single intensity value per direction (azimuthal and elevation angle), it cannot distinguish two objects that lie at different depths along the same direction to the receiver. Wision extracts this depth information by performing beamforming at the wireless transmitter. Intuitively, beamforming changes the region of space that is illuminated by wireless signals and hence enables the receiver to focus on reflections from different depths along the same azimuthal and elevation angles. Thus, a Wision receiver computes the intensity values for different transmit beamforming directions and stitches them together to image objects that lie along different depths. In the rest of the paper, we expand on the above idea and demonstrate how to image objects in both line-of-sight and non-line-ofsight scenarios. We also extend our approach to work with one-dimensional antenna arrays and describe our algorithm to combine images across multiple wireless transmitters.

Wision is related to recent work on motion detection using Wi-Fi Doppler [14, 38, 19]. These systems extract the minute changes in the Wi-Fi signals caused by human motion such as running [14], walking forward and backward [19], and gestures [38]. While we build on this work, Wision is the first system that leverages narrowband signal (e.g., Wi-Fi) reflections to create images of static objects in the environment.
To show the feasibility of our approach, we implement a Wision receiver on USRP-N210s operating at $2.4 \mathrm{GHz}$ using the phase and amplitude of the first OFDM data sub-carrier. We evaluate the effects of various properties of objects, including size, material, and orientation, on their interaction with Wi-Fi signals. We present imaging results using various receiver configurations ranging from $4 \times 1$ to $8 \times 8$ antenna arrays. We experiment in various line-of-sight and non-lineof-sight scenarios where the object is separated from both the transmitter and the receiver. Our results show the following:

- Using two-dimensional antenna arrays, Wision could create images of a metallic T-shaped structure, similar to Fig. 2. The object's length and height in the resulting image are within 5 and $8 \mathrm{~cm}$, and 18 and $40 \mathrm{~cm}$ of the actual object, using $8 \times 8$ and $4 \times 4$ antenna arrays respectively.

- Wision's imaging capability extends to common objects such as leather couches and in non-line-of-sight scenarios where the object is separated from the transmitter and the receiver by a barrier $4 \mathrm{~cm}$ thick.

- The imaging capability however is limited by the material of the object: metallic surfaces reflect better than wooden surfaces, which in turn reflect better than foam. Also, the imaging ability reduces with the object's size: objects that are smaller or comparable to the wavelength of the wireless signals have very weak interactions making them difficult to image. Finally, the blurriness of the images (as seen in Fig. 2) is dependent on the signal wavelength and is inherent to imaging using Wi-Fi signals.

We also perform feasibility studies of three applications: static human localization, object localization, and throughthe-fabric detection of metallic objects. Our results show the following about Wision:

- It localizes static humans with a median accuracy of $26 \mathrm{~cm}$, even in the presence of motion from other humans in the environment.

- It localizes a HP desktop with a median accuracy of $15 \mathrm{~cm}$, in an office environment with other furniture.

- It could detect a passive MacBook Pro placed in a backpack and a passive smartphone placed in the pocket of a hoodie worn by a human subject.

Contributions: We explore the feasibility of imaging using narrow band signals (e.g., $\mathrm{Wi}-\mathrm{Fi}$ ) by leveraging the multipath reflections. To do this, we show how to extract the intensities of the wireless reflections from different azimuthal and elevation angles by leveraging multiple-antennas at the AP. We also introduce mechanisms that extract the depth information using transmit beamforming. We build a prototype and demonstrate its ability to create images in both line-ofsight and non-line-of-sight scenarios. Finally, we discuss the limits of our Wi-Fi based approach to imaging.

\section{Related Work}

Our work is related to prior art in two domains.

(a) Wi-Fi Systems: The related work in this domain falls in two main areas: Wi-Fi localization and device-free motion detection. There has been significant work on Wi-Fi device localization [8, 53] using techniques including RSSI [15, 48] and fine-grained channel state information [44]. The state of the art systems [7, 21, 51] achieve sub-meter localiza- 
tion accuracies using statistical angle-of-arrival techniques such as MUSIC [43]. More recently work on motion detection [38, 19, 28, 14, 42, 46] uses changes to the wireless signals caused by human motion such as running [14], walking forward and backward [19], body gestures [38, 28], and activity recognition [42, 46]. Wision goes beyond these device localization and motion detection systems and enables imaging of static objects and humans.

(b) RF Image Sensing: Traditional radar systems have been used to perform human motion and activity detection [4, 40, 31, 34, 5, 18, 29] and detection of static metallic objects [13, 39, 49, 55, 27]. These systems however use expensive ultra-wideband transceivers, multiple antennas, and specialized signal modulation (e.g., FMCW [32]). Specifically, [13, 39, 49] use $500 \mathrm{MHz}$ to $3 \mathrm{GHz}$ wideband transmissions to detect human motion and image metallic objects. Wision instead performs imaging of objects using narrowband wireless transmissions (e.g., Wi-Fi). Further, Wision achieves this using the channel amplitude and phase information from Wi-Fi OFDM transmissions, instead of requiring special modulated signals (e.g., FMCW).

Wision is also related to recent proposals [10, 35] that use techniques like compressive sensing with single-antenna devices and full-duplex radios to detect the presence of any object in a sparse environment. In contrast, Wision addresses the more general problem of transforming Wi-Fi into a camera that creates images of objects. We also build a prototype of Wision and show that it can image and localize objects and humans in line-of-sight and non-line-of-sight scenarios.

Wision also builds on foundation work on radio tomography imaging [54, 50, 37]. These systems track human motion including fine-grained breathing motion by deploying ten to thirty sensors spread throughout the environment and measuring the channel attenuation between every pair of sensors. Recent work also creates images of objects using robotic Wi-Fi transmitter-receiver pairs that move around the object of interest to measure its absorption properties [36, 23, 24]. Wision differs from this line of work in two ways: First, our goal is to use static Wi-Fi infrastructure to enable sensing and imaging applications using few Wi-Fi transmitters, without the need for multiple sensors or robotic deployments. To achieve this we leverage multiple antennas to extract the object reflections in the environment. Second, tomography systems [36, 24] use wireless absorption properties of the objects for imaging. In contrast, we use wireless reflections and scattering to perform imaging, and hence require a different set of algorithms.

Finally, our work builds on prior work in MRI, optical, microscopy, and computational photography. In contrast to this work, we focus on exploring the feasibility of imaging using OFDM based signals that are commonly used in wireless communication systems (e.g., Wi-Fi).

\section{Wision Overview}

Wision is a novel approach to imaging using narrowband wireless signals (e.g., Wi-Fi). It leverages transmissions from Wi-Fi devices in the environment to perform imaging on the AP. Wireless transmissions experience multi-path reflections that effectively "light up" reflective objects in the

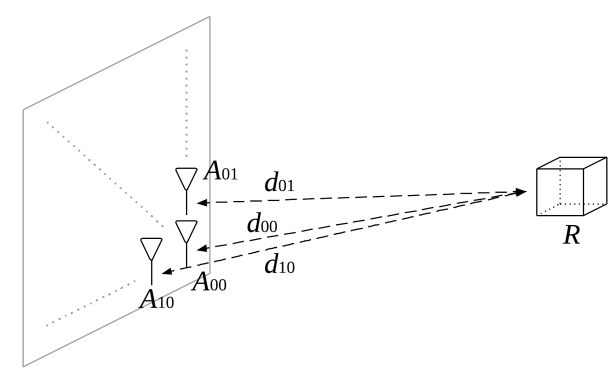

Figure 3-Overview of Imaging Radar Approaches. The transmitter sends a very narrow pulse and the receiver computes the intensity of the region $R$ by measuring the pulse echoes from distances of $d_{00} \cdots d_{n n}$ at the antenna elements. This requires ultra-wideband transmissions that is not applicable to Wi-Fi signals.

environment, which the Wision receiver uses to image the objects. Wision leverages multiple antennas at the receiver and is designed to be implemented on a Wi-Fi AP. The transmitters can be either mobile devices, like smartphones and laptops, or other APs on the same Wi-Fi channel. In the rest of this section, we first provide an overview of imaging radar approaches. We then describe the advantages and challenges of using Wi-Fi over radar imaging.

\subsection{Overview of Radar Approaches}

Radar systems operate by transmitting a short narrow pulse and waiting for the pulse to hit an object and return back. They then compute the time difference between the two events to estimate the distance from the object. From radar theory, to distinguish two objects that are separated by $\Delta d$, the duration of the pulse, $\Delta p$, must satisfy [47]:

$$
\Delta p \leq \frac{2 \Delta d}{c_{0}}
$$

where $c_{0}$ is the speed of the wireless signals. The above equation states that to distinguish two close-by objects, the length of the pulse must be short. This is expected because the echoes of longer pulses merge together and make it difficult to identify the echoes as two separate objects. Since shorter pulses occupy wider bandwidth, radar approaches require a large bandwidth to distinguish objects in the environment. For instance, achieving a resolution of $50 \mathrm{~cm}$ requires a transmission bandwidth of $300 \mathrm{MHz}$.

Imaging radar systems (e.g., SAR [20, 12]) leverage the above approach to create an image. Specifically, these systems use antenna arrays (or equivalently, moving antennas) as the receiver, as shown in Fig. 3 . To compute the intensity of a particular region $R$, the receiver measures the pulse echoes on each of the antennas from distances of $d_{00}, \cdots, d_{n n}$, as shown in the figure. The receiver then coherently combines these echoes across all its antennas to compute an intensity value for $R$. By repeating this procedure across regions, radar systems create an image of the space.

Note that the resolution of the radar imaging is determined by its ability to compute accurate distance measurements from each of the receive antennas; this requires narrow pulses that use ultra-wideband transmissions. We note that many practical radar systems use specialized signals (e.g., 
FMCW [32, 39]); these systems however still need similar ultra-wideband transmissions to perform imaging.

\subsection{A Case for Using Wi-Fi over Radar Imag- ing}

Using Wi-Fi over radar for imaging is attractive for two main reasons: First, the cost of a Wi-Fi chipset is significantly lower than a radar device given its economy of scale and lower bandwidth requirements. Thus, a Wi-Fi imaging solution would enable imaging capabilities at a much lower cost than radar-based solutions. Second, for the same power level, mono-static radar systems (where the transmitter and the receiver are co-located) have a much lower range of operation than bi-static radar systems (where the transmitter and receiver are separated in distance) [38]. Thus to achieve a good range (e.g., a whole-home coverage), the radar transmitter-receiver pair should be separated and deployed at different locations. Further, reliable wireless imaging requires deploying multiple radar transmitters at various angles with respect to the object. In contrast, Wi-Fi devices are already spread out in the environment, and hence naturally provide a good imaging range as well as light the object from various angles to provide reliable imaging.

\subsection{Challenges Using Wi-Fi for Imaging}

Wi-Fi transmissions impose significant constraints that make it challenging to leverage radar imaging approaches:

- Wi-Fi has orders of magnitude lower bandwidth than is required for radar imaging. Typical Wi-Fi transmissions occupy a bandwidth of $20 \mathrm{MHz}$; a radar system with a $20 \mathrm{MHz}$ bandwidth provides a maximum resolution of 7.5 meters, which is inadequate for imaging purposes. We also note that while the above distance-based radar systems use multiple antennas, they require accurate computation of the distance values from an object of interest to each of its antennas.

- Wi-Fi signals are OFDM-based transmissions that are not designed for imaging purposes. Our goal is to leverage transmissions from Wi-Fi devices in the environment. Thus, we cannot use custom radar signal waveforms including narrow pulse transmissions and FMCW signals We also note that the channel state information [25] is increasingly becoming accessible on Wi-Fi devices; thus, it is desirable to have an imaging solution that uses only the channel amplitude and phase information.

\section{Wision Design}

To avoid the above challenges, Wision creates images using a different principle than that of radar systems. Wision's approach is similar in spirit to optical imaging systems where images are typically formed by measuring the incoming signal intensities at each azimuth and elevation angle. This avoids the need for distance computation and hence is not constrained by the requirements of traditional radar systems.

Achieving this in practice is, however, not straightforward because the receiver receives the combination of reflections from multiple regions in space on each of its antennas. In a

\footnotetext{
${ }^{1}$ In contrast to Wi-Fi that uses OFDM, FMCW signals use hundreds of megahertz/GHz bandwidth and sweep the frequency across time; pulse radar signals send very narrow pulse in time.
}

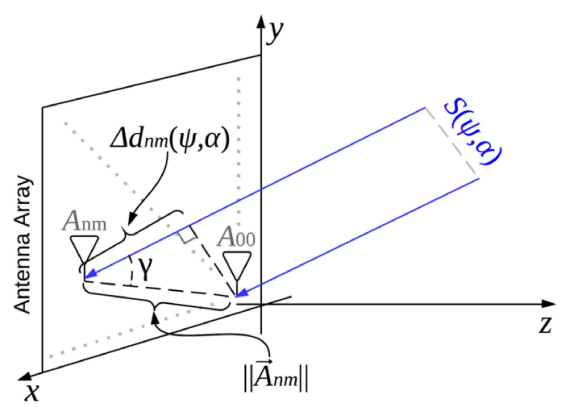

Figure 4-Calculating the phase difference between antenna $A_{n m}$ and $A_{00}$. For the signal $S(\psi, \alpha)$, the difference in the received phase between $A_{n m}$ and $A_{00}$ is related to the additional distance $\Delta d_{n m}(\psi, \alpha)$ that $S(\psi, \alpha)$ travelled to reach $A_{n m}$.

optical system, a lens is used to physically separate the received signals from different directions. Wision, in contrast, uses multiple antennas and Fourier analysis to separate these signals. In the rest of this section, we first describe our image construction algorithm using a two-dimensional antenna array at the receiver. We then extend our design to work with single-antenna arrays. Next, we describe how we combine images across multiple transmitters. Finally, we outline our algorithm to extract depth using transmitter beamforming.

\subsection{Wision's Imaging Algorithm}

As shown in Fig.4, Wision performs imaging using multiple antennas at the Wi-Fi receiver. We first show that the signal from each azimuthal and elevation angle corresponds to a specific pattern of phase differences (i.e., a basis function) across the antennas. We then describe how Wision extracts the multi-path intensities at different azimuthal and elevation angles by performing a Fourier transform.

\subsubsection{Mapping directions to basis functions}

The received signal is a linear combination of the multipath reflections from different directions (i.e., azimuthal and elevation angles). Consider the multi-path reflection, $S(\psi, \alpha)$, arriving at the receiver from an azimuthal angle $\psi$ and an elevation angle $\alpha$. These reflections correspond to specific phase differences across the antennas at the receiver.

To see this, consider the antenna array along the x-y plane, Fig. 4, where the antenna, $A_{00}$, is located at the origin. Say that the antennas are each separated by $d$ and that there are a total of $N$ and $M$ antennas along the x-axis and y-axis respectively. We compute the phase difference between the antenna-pair, $A_{n m}$ and $A_{00}$. From basic physics, the phase change in a complex wave as it traverses a distance $\Delta d$ is given by $e^{j \frac{2 \pi \Delta d}{\lambda}}$, where $\lambda$ is the signal wavelength. Thus, the phase change, experienced by the signal $S(\psi, \alpha)$ is given by:

$$
e^{j \frac{2 \pi \Delta d_{n m}(\psi, \alpha)}{\lambda}}
$$

where $\Delta d_{n m}(\psi, \alpha)$ is the extra distance traversed by the signal between $A_{00}$ and $A_{n m}$, as shown in the figure. Using trigonometric identities, we derive the following equations that are 


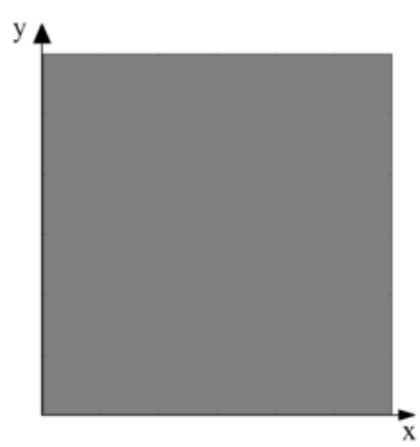

(a) $\psi=0^{\circ}, \alpha=0^{\circ}$

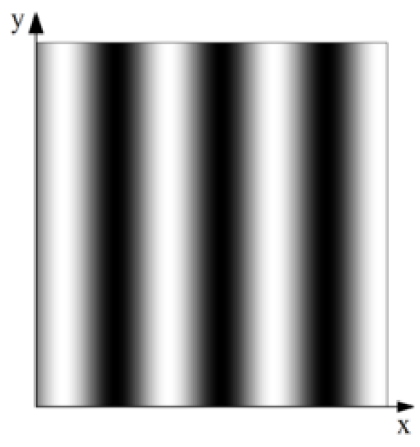

(b) $\psi=30^{\circ}, \alpha=0^{\circ}$

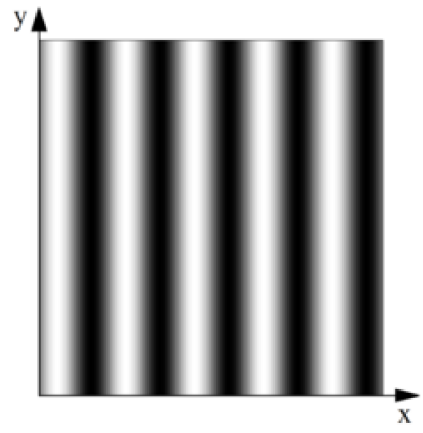

(c) $\psi=60^{\circ}, \alpha=0^{\circ}$

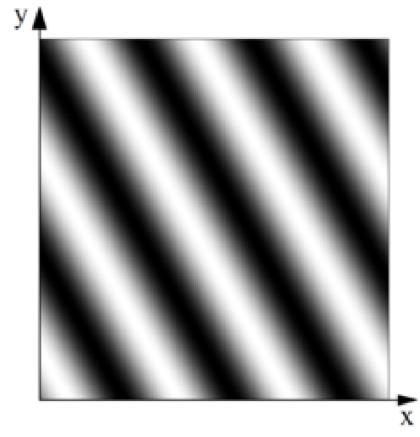

(d) $\psi=60^{\circ}, \alpha=30^{\circ}$

Figure 5-Basis functions for various azimuthal $(\psi)$ and elevation angles $(\alpha)$. Signals incoming from different azimuthal and elevation angles correspond to basis functions of differing frequencies and orientations. Treating the incoming signals as a combination of basis functions enables efficient operations, such as the 2D-FFT, to be performed upon them.

satisfied in the figure:

$$
\begin{gathered}
\Delta d_{n m}(\psi, \alpha)=\left\|\vec{A}_{n m}\right\| \cos (\gamma) \\
\cos (\gamma)=\frac{S(\psi \vec{\psi}, \alpha) \cdot \vec{A}_{n m}}{\|S(\vec{\psi}, \alpha)\|\left\|\vec{A}_{n m}\right\|}
\end{gathered}
$$

where $\vec{A}_{n m}$ is the vector from the origin to the antenna element $A_{n m}, \vec{S}(\psi, \alpha)$ is the vector corresponding to the signal, $S(\psi, \alpha)$, and the $(\cdot)$ operation computes the dot product between two vectors. Now, given that the antenna array $A_{n m}$ is at position $(n d, m d, 0)$, where $d$ is the distance between the antennas, $\vec{A}_{n m}$ can be written as,

$$
\vec{A}_{n m}=[n d, m d, 0]^{T}
$$

Similarly, since the signal $S(\psi, \alpha)$ arrives at the receiver along the azimuthal angle $\psi$ and elevation angle $\alpha$, we have the unit vector corresponding to the signal $S(\psi, \alpha)$ as,

$$
\frac{\vec{S}(\psi, \alpha)}{\|\vec{S}(\psi, \alpha)\|}=[\cos (\alpha) \cos (\psi), \sin (\alpha), \cos (\alpha) \sin (\psi)]^{T}
$$

Combining all the above equations, we can rewrite the phase change between antennas, $A_{n m}$ and $A_{00}$ in Eq. 1 as,

$$
e^{j(n d \cos (\alpha) \cos (\psi)+m d \sin (\alpha))}
$$

Now if we define the basis function of the signal arriving from an azimuthal angle $\psi$ and elevation angle $\alpha, B_{\psi, \alpha}$, as the pattern of phase differences from all the antennas to $A_{00}$, we can write the basis function as the following matrix:

$$
B_{\psi, \alpha}=\left[e^{j(n d \cos (\alpha) \cos (\psi)+m d \sin (\alpha))}\right]_{n, m}
$$

The above equation states that the basis function changes with the azimuthal and elevation angles, which we show in Fig. 5 The plots show that there is a distinct pattern that corresponds to each angle between 0 and 180 degrees. In the next section, we will use these patterns to extract the intensity of the multi-path reflections from different azimuthal and elevation angles. We note that to differentiate between objects throughout 360 degrees, we can place one of the antennas in a different plane than the rest of the antenna array.

\subsubsection{Extracting images from basis functions}

The above description shows that the received signal can be decomposed into a linear combination of the basis functions, each of which arrives from a specific azimuthal and elevation angle. Wision creates an image by extracting the intensity values corresponding to each basis function. At a high level, given that each azimuthal and elevation angle corresponds to a specific basis function, we get the intensities by correlating with the corresponding basis functions. Specifically, to get the intensity at azimuthal and elevation angles of $\psi$ and $\alpha$, one can compute the following summation:

$$
\begin{aligned}
I(\psi, \alpha) & =\sum_{n=0}^{N-1} \sum_{m=0}^{M-1} x(n, m) B_{\psi, \alpha}(n, m) \\
& =\sum_{n=0}^{N-1} \sum_{m=0}^{M-1} x(n, m) e^{-j d(n(\cos (\alpha) \cos (\psi))+m(\sin (\alpha)))}
\end{aligned}
$$

where $x(n, m)$ is the received signal on the antenna element $(n, m)$ and $B_{\psi, \alpha}$ is the basis function matrix corresponding to the azimuthal angle $\psi$ and elevation angle $\alpha$. Intuitively, the above equation adjusts the phase of the received signal to correspond to that of the basis function. This ensures that only signals arriving from a specific azimuthal and elevation angle would coherently combine, while all the remaining signals combine non-coherently. Taking the summation over all antenna elements ensures that the desired signals are amplified, hence giving us the intensity value for a specific azimuthal and elevation angle.

We note that the above computation is identical to a twodimensional Fourier transform. Specifically, the equation for a 2-D Fourier transform given a two-dimensional input signal $x(n, m)$, is given by,

$$
X(u, v)=\frac{1}{N M} \sum_{n=0}^{N-1} \sum_{m=0}^{M-1} x(n, m) e^{-j \Omega(n u+m v)}
$$

where $\Omega$ is the fundamental Fourier frequency [17]. We note that the above two equations are identical to a constant factor, when we substitute $u$ for $\cos (\alpha) \cos (\psi)$ and $v$ for $\sin (\alpha)$. Thus, a two-dimensional Fourier transform directly provides us with the intensity values for each azimuthal and elevation angle. Given this property, we summarize our algorithm: 
Initialization. The receiver measures the phase and magnitude information from all of its antennas. Note that phase and magnitude information are both easily obtained from channel state information (CSI) that is increasingly available on commodity Wi-Fi hardware [25].

Step 1. Compute the azimuthal $(\psi)$ and elevation $(\alpha)$ angles for a given region in the space.

Step 2. Compute the corresponding intensity value by performing a 2D Fourier transform operation and mapping $u$ and $v$ in the 2D-FFT equation to $\cos (\alpha) \cos (\psi)$ and $\sin (\alpha)$.

Step 3. Repeat Step 1 and Step 2 for every region in the twodimensional space to generate images with proportions that are visually expected by the human eye.

\subsection{Wision with One-Dimensional Antenna Arrays}

The discussion so far has focused on using a twodimensional antenna array at the Wi-Fi receiver. Wision, however, applies even if the receiver only has a onedimensional antenna array. Here, the receiver cannot distinguish between different elevation angles. However, given an incoming signal at an angle $\psi$, the antenna array sees a phase change that maps to a particular frequency in the Fourier transform. Specifically, for an incoming angle $\psi$, the difference in the phase seen between the first antenna and the $n^{\text {th }}$ antenna can be written as $\Delta \psi(n)=\frac{2 \pi(n-1) d \cos (\psi)}{\lambda}$, where $\lambda$ is the signal wavelength, and $d$ is the distance between adjacent antennas in the array. Thus, the signals incoming from different angles $\psi$, corresponds to one-dimensional cosine functions of different frequencies. These cosine functions form a set of one-dimensional basis functions; Wision can perform a one-dimensional fast Fourier transform to determine the weight of each of these basis functions, and thereby determine the intensity of the reflections incoming from each direction. We note that the resulting images visually represent the intensity of signals incoming from different directions. Such a representation does not provide the elevation information and hence is not as intuitive to the human eye. However, they can still be used to detect the presence of reflective objects at various angles from the receiver. We evaluate this property in $\$ 7.1$

\subsection{Accounting for the Wi-Fi transmitter}

So far we have focused on receiving the multi-path signals from a transmitter that get reflected off of objects. In practice, however, there might be a strong direct signal from the transmitter. In this section, we first describe how Wision cancels these direct signals from the Wi-Fi transmitter. We then show how Wision combines transmissions from multiple Wi-Fi devices to enhance the images.

\subsubsection{Removing direct Wi-Fi transmissions}

The direct path from a Wi-Fi transmitter to the receiver can have significantly higher energy than the reflections off objects in the environment. Fig. 6 (a) shows the image captured by a one-dimensional antenna array of a metal bottle with diameter $8 \mathrm{~cm}$ and height $25 \mathrm{~cm}$. We place the transmitter and the object at angles 20 and 60 degrees respectively from the receiver. The figure shows that the direct transmitter signal is stronger than the bottle's reflection.

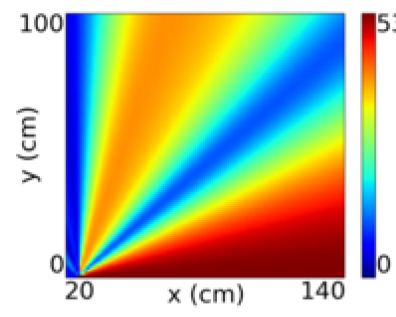

(a) Bottle before nulling

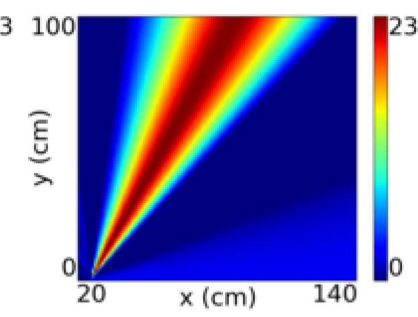

(b) Bottle after nulling
Figure 6-Removing direct Wi-Fi transmissions. The figure shows the effect of nulling the direct Wi-Fi transmissions. The reflections corresponding to the metallic water bottle were weaker before the nulling operation. The bottle can be seen with a higher contrast after the nulling operation.

Wision addresses this problem by leveraging the multiple antennas at the receiver to null out the signal in the direction of the Wi-Fi transmitter using interference nulling [33]. Fig. G(b) shows the result of this operation; the object can now be seen with a higher contrast than before. We note the following key points about the above design.

- The nulling approach can also be extended to work with multiple objects and walls. Specifically, similar to nulling the direct path from the transmitter, the receiver can null the reflections from a strong-reflective object (e.g., metal), to improve the visibility of weak-reflective objects (e.g., plastic). The resolution with which we can achieve this depends on the number of bits at the ADC; our experiments use 10 bits at the ADC.

- The nulling operation eliminates the ability to image objects that lie along the same azimuthal and elevation angles as the transmitter. We can however address this problem by combining information across two Wi-Fi transmitters at different locations, which we describe next.

\subsubsection{Using multiple transmitters}

In principle, the Wision receiver can create images using transmissions from every Wi-Fi device in the environment, and combine the images to achieve a more reliable mechanism. Since Wi-Fi devices use carrier sense and they transmit at different times, their transmissions do not interfere with each other and hence the receiver can independently create images for each transmitter. However, Wi-Fi devices can vary in various aspects including transmission power, antenna gains, and their orientations. Thus, naively summing up the intensity values across transmitters is not desirable. Wision instead combines the images after normalizing each image with the maximum intensity observed in it. This accounts for differences that are inherent to wireless devices.

\subsection{Extracting depth information}

Since the above algorithm produces a single intensity value for each azimuthal and elevation angle, it can neither identify an object's depth nor differentiate between objects that lie along the same direction from the receiver.

Wision addresses this problem by performing beamforming at the transmitter (see Fig. 7). Specifically, if two objects lie at different depths but at the same azimuthal $\psi$ and elevation $\alpha$ angle relative to the receiver, then Wision can identify and distinguish them by focusing its transmitter's signals at different depths of the receiver. When the transmitted sig- 


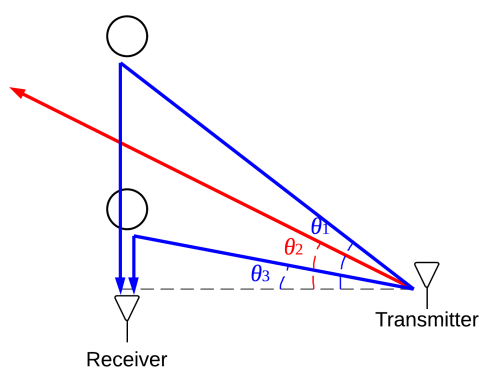

Figure 7-Wision beamforming. Wision can use transmitter beamforming to distinguish objects along the same azimuthal and elevation angle. In contrast, mono-static radars need to use ultra-wide band transmissions to distinguish such objects.

nal is directed at an object, the receiver sees a strong signal incoming from $\psi$ and $\alpha$. If the signal is not directed at any specific object, then there will not be a strong reflection. This allows Wision to identify the depth of each object.

More generally, our algorithm works by having the transmitter beamform at different angles with respect to itself. The receiver computes the intensities for all the azimuthal and elevation angles that intersect with the transmitter's beamforming direction. By stitching together the intensity values for different beamforming directions, Wision can reconstruct the intensity values at different positions that lie along the same azimuthal and elevation angles from the receiver, allowing it to extract the depth information.

The above approach is possible because Wision leverages Wi-Fi transmitters that are not co-located with the receiver. Specifically, in traditional mono-static radar systems, the transmitter and receiver are co-located. Therefore, the only angular parameters that can be changed are the azimuthal and elevation angles with respect to the radar device. As a result, to image objects at different positions along the same direction, these systems have to use ultra-wide band transmissions. In contrast, in Wision, the transmitter and the receiver are at different positions. Thus, we have three free parameters, the azimuthal and elevation angles at the receiver, and the beamforming angle, $\theta$, at the transmitter, which we vary to image at different depths with respect to the receiver.

In practice one can achieve transmit beamforming using MIMO pre-coding, phased arrays, and directional antennas. Transmit beamforming can be implemented on other APs in the network that are likely to have multiple antennas. We note that the effectiveness of beamforming depends on the transmit beam width that in turn depends on the number of antennas (for multi-antenna systems) and the directionality coefficients (for directional antennas). We use a directional antenna with a coarse directionality of 16 degrees to demonstrate the feasibility of our approach. One can, however, achieve much better results by using either MIMO precoding or better directional antennas.

\section{Wi-Fi Interaction with Objects}

Objects either reflect or absorb Wi-Fi signals, or allow Wi-Fi signals to pass-through them. While Wision leverages the pass-through property of objects to enable imaging in non-line-of-sight and through-the-wall scenarios, Wision mainly uses their reflective property to achieve imaging. In this section we describe three aspects that determine the interaction of $\mathrm{Wi}-\mathrm{Fi}$ signals with objects in the environment.

(a) Material of the object. A metallic surface reflects a large portion of the incident wireless signals, while these signals mostly pass-through commodity plastic surfaces. Thus the material of an object affects its reflective properties. Further, objects also differ in the type of reflection they possess. Specifically, reflection can be broadly classified as either diffuse reflection or specular reflection [9]. In diffuse reflection, the object scatters the incident signals in all the directions. In specular reflection, the object acts like a mirror while following the laws of reflection, i.e., the angle of reflection is such that a line drawn perpendicular to the surface bisects the angle between the reflected and incident waves. Every object has both diffuse and specular reflection properties to varying extents depending on the smoothness of the reflection surface - smooth surfaces in general have a better specular reflection property than rough surfaces. While one can design algorithms to identify the material given its reflective properties, this is not in the scope of this paper.

(b) Size of the object. Smaller objects of the same material have smaller cross-sections and hence reflect a smaller fraction of the incident wireless signals. Further, as the size of the object becomes proportional to the wavelength of $\mathrm{Wi}-\mathrm{Fi}$ signals (approx. $12 \mathrm{~cm}$ at $2.4 \mathrm{GHz}$ ), its interaction with the Wi-Fi signals reduces. Our results confirm this hypothesis. We however note that using the nulling mechanism in $\$ 4.3$ our prototype implementation could identify a metallic pole with diameter less than $2 \mathrm{~cm}$, albeit with lower intensity.

(c) Diffraction effects. Finally, the images generated by Wision are blurry in nature. This is a result of diffraction (i.e., interference) effects of the Wi-Fi signals, which limits our angular resolution. Specifically, because of diffraction, instead of seeing a source from a particular azimuthal and elevation angle as a point, we see the source as an Airy disk pattern [6], which in traditional imaging are concentric circles of constructive and destructive interference. As the Airy disk patterns from different signal sources overlap and merge, they create the blurriness seen in Wision's images. The extent of diffraction can be quantified by the Rayleigh criterion [26], which states that the angular resolution $\theta$ is:

$$
\theta \approx 1.220 \frac{\lambda}{D}
$$

where $\lambda$ is the wavelength of the signals used, and $D$ is the horizontal or vertical length of Wision's antenna array. Thus, in general, we can reduce the blurriness in the images by either using higher frequency transmissions (e.g., $5 \mathrm{GHz}$ versus $2.4 \mathrm{GHz}$ ) or by increasing the length of the antenna array.

While the above discussion is not a comprehensive list of all the properties that effect Wi-Fi's interaction with objects, it helps us better understand the images in our experiments.

\section{Implementation}

We implement a prototype of Wision on the software radio platform and evaluate it on the USRP-N210 hardware. We note that Wision works using only the CSI information (i.e., amplitude and phase in each OFDM subcarrier, per an- 


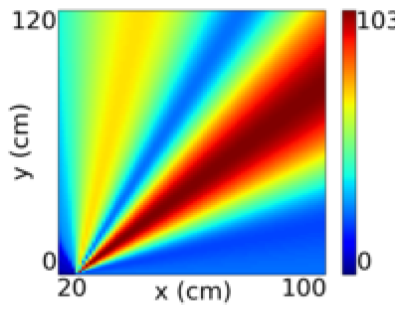

(a) $18^{\circ}$

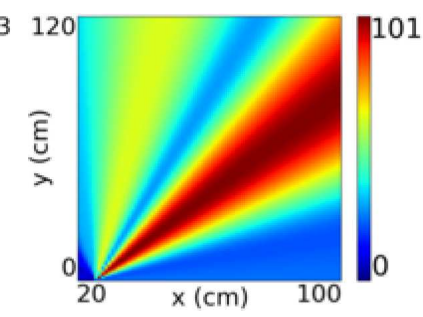

(b) $15^{\circ}$
Figure 8-Wision with two objects placed at different angles from the receiver. The figure shows that Wision creates distinguishable streaks when the angle between the objects is greater than 15 degrees. This resolution can be reduced to 7.5 degrees by using $5 \mathrm{GHz}$ Wi-Fi.

tenna) that is provided by Intel 802.11 n chipsets [25]. However, extracting the CSI information from 802.11ac chipsets with more than three antennas requires purchasing expensive (from a cost perspective) firmware updates from the chipset manufacturers. Thus, to evaluate Wision we replicate a WiFi OFDM receiver on the USRP hardware with 54 subcarriers and random data payload. Specifically, each USRP is fitted with a XCVR2450 daughter board that enables reception of OFDM signals at the $2.4 \mathrm{GHz}$ frequency range using a $10 \mathrm{MHz}$ bandwidth. We use only $10 \mathrm{MHz}$ since it is difficult for USRPs to reliably receive the I/Q samples in software at $20 \mathrm{MHz}$. We use the amplitude and phase on the first OFDM data sub-carrier to run our imaging algorithms. In principle, one can improve the reliability by combining the resulting images from all the OFDM data sub-carriers; this is however not in the scope of this paper. We run experiments with $8 \times 8,4 \times 4,8 \times 1$, and $4 \times 1$ antenna arrays. Instead of using 64 USRP-N210s to build an $8 \times 8$ antenna array which would be expensive, we move an USRP-N210 on a linear actuator and sample the transmitted signal at eight different locations separated by half a wavelength. We then move the linear actuator to eight different heights, each separated by half a wavelength. We note that using linear actuators is a standard technique for evaluating antenna arrays [41, 16].

We create images using both one-dimensional and twodimensional antenna arrays as our receiver. The images created by one-dimensional antenna arrays show the intensities of the multi-path reflections along different angles to the receiver. The two-dimensional antenna array images, on the other hand, show the reflection intensities as a function of the width and the height of the target area. Finally, to perform beamforming, we use a log periodic directional antenna [2] at the transmitter that is rotated over 180 degrees in steps of ten degrees. We then stitch together the receiver measurements across the transmit beamform directions to create an intensity map as a function of the depth.

\section{Evaluation}

We evaluate our prototype in an office building. First, we use our one-dimensional antenna arrays to evaluate the ability to identify objects at different angles and depths with respect to our prototype receiver. Next, we demonstrate Wision's ability to image objects in line-of-sight and non-lineof-sight scenarios using 2-D antenna arrays.

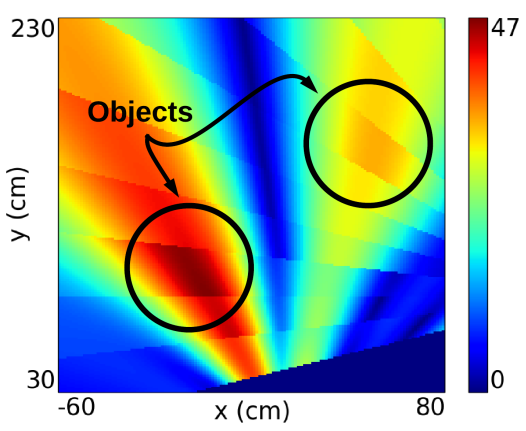

Figure 9-Depth of two objects at different azimuthal and elevation angles relative to the receiver. The figure shows the intensity values stitched together from different transmit beamforming directions. The depth of the objects corresponds to the regions of high intensity in the plot. The streaks of intensity are due to the coarse directionality of our antennas.

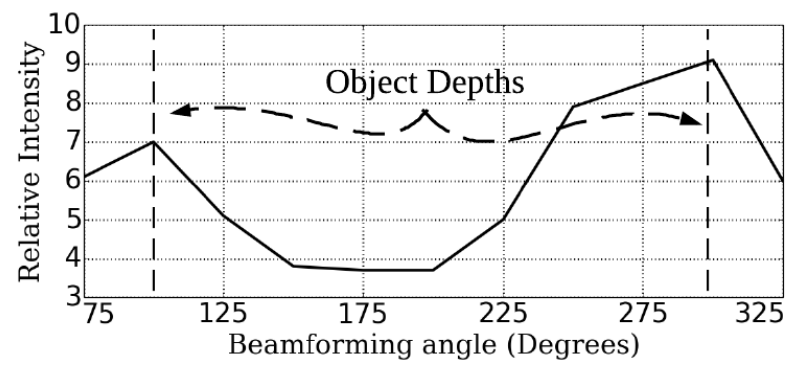

Figure 10 - Depth of two objects at the same azimuthal and elevation angle relative to the receiver. We plot the intensity values along the direction of the objects as a function of depth. The figure shows that the areas of high intensity can identify the depth of both the objects.

\subsection{Imaging Using 1-D Antenna Arrays}

We evaluate Wision's ability to image two objects using our 1-D antenna array receiver. We first image objects at different directions from the receiver, and then image objects that lie along different depths from the receiver.

\subsubsection{Objects at different receiver directions}

We run experiments with two metallic objects of dimensions $16 \times 20 \times 5 \mathrm{~cm}^{3}$ placed at two different angles to the receiver. Specifically, we place our $8 \times 1$ array receiver and the single-antenna transmitter at coordinates $(0,0)$ and $(75,60)$ respectively. We place the objects at different angles from the receiver. We repeat the experiments as we move the objects closer to each other. Fig. 8 plots the results for two different angles between the two objects. The plots show that when the angle between the objects is 15 degrees or greater, the receiver sees two distinct streaks corresponding to the two objects. We note that our receiver's angular resolution improves at higher frequencies — the above resolution can be further reduced by using $5 \mathrm{GHz}$ Wi-Fi signals.

\subsubsection{Objects at different receiver depths}

As described in $\$ 4.4$ Wision has the ability to extract the depth information of each object and also identify hidden objects that lie along the same direction. We evaluate both these aspects in this section.

Experiment 1. We place two metal desktops $(44 \times 44 \times 18$ $\mathrm{cm}^{3}$ ) at angles of 80 and 130 degrees from the receiver. The first object is placed at a depth of two meters and the second 
object is placed at a depth of 0.8 meters. Our goal in this experiment is to extract the depth values of both these objects using the algorithm described in $\$ 4.4$. To do this, we place an $8 \times 1$ receiver antenna array and a single-antenna transmitter at coordinates of $(0,0)$ and $(80,80)$ respectively. The transmitter employs the log-directional antenna [2] to beamform signals along different depths. The receiver stitches together the intensity values at different depths to create a 2D-image as a function of the depth and the width.

Fig. 9 plots the image created by the above experiment. The figure shows two bright intensity blobs centered at depths of 2 meters and 0.8 meters, which corresponds to the positions of the two objects. We note that since the directional antenna we use has a very coarse directionality, we see reflections with moderate-energy along all depths for each object's direction. We note however that the intensities are the strongest at the positions corresponding to the objects.

Experiment 2. We place two objects one behind the other along the same direction to the receiver. The object in the front (a stack of books) is placed to hide the object behind it (a laptop); they are at depths of 1 and 3 meters respectively. We use an eight-antenna array as our receiver and a HG2415G directional grid antenna [1] at the transmitter. The receiver stitches together the intensities along the direction of the objects at various depths using our depth algorithm.

Fig. 10 shows the reflection intensities as a function of depth. The plot shows two peaks corresponding to the depth values of the objects. We also see a higher reflection intensity for the object in the back. This is because the object in the back is a metallic object that has a much higher reflective intensity than a stack of books.

Observed Limitations: We note two key observations from our experiments. First, the object's orientation with respect to the transmitter and the receiver was a key factor in determining the resulting image quality. Specifically, to identify the object in the image, the transmitter's signals must effectively light up the object and further the reflected signals must reach the receiver. To address this issue, one could optimize the antenna position, use antennas with better radiation patterns or combine images taken from multiple Wi-Fi devices in different locations. Second, in a setting with objects of different materials like metal, wood and plastic, a strong reflector (e.g., flat metallic surface) would appear predominantly in an image. Our ability to distinguish a weaker reflecting object in the presence of such a strong reflector is dependent on the amount of nulling we can achieve. With our current nulling capability of $28-30 \mathrm{~dB}$, when two objects are placed behind each other, a strong reflector (e.g., a large metallic object) in the front could in certain receiver angles obstruct reflections from a weak reflector behind it. However, we believe that with better nulling capabilities [11, 10], one can improve our ability to image weak reflectors.

\subsection{Imaging using 2D Arrays}

As described in $\$$, imaging using two-dimensional antenna arrays creates an intensity map that is a function of width and height. To see this, we perform imaging on a metallic T-structure with dimensions $44 \times 62 \times 44 \mathrm{~cm}^{3}$ that is placed 1.2 meters away from the receiver. We run experi-

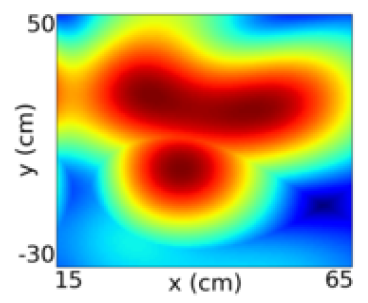

(a) $8 \times 8$

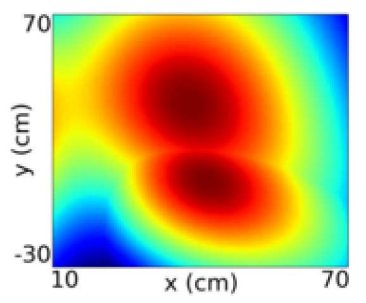

(b) $4 \times 4$
Figure 11-Imaging a metal T-structure. (a) shows an image captured using an $8 \times 8$ antenna array, and (b) shows an image captured using a $4 \times 4$ antenna array. The length and height of the object in the resulting image are within 5 and $8 \mathrm{~cm}$ of the actual object using the $8 \times 8$ antenna array and 18 and $40 \mathrm{~cm}$ using a $4 \times 4$ antenna array. This is in comparison to the 15 meter resolution using FMCW radar systems with a comparable bandwidth.

ments with single-antenna transmitters and an $8 \times 8$ antenna array as a receiver. The transmitter and receiver are fitted with omni-directional antennas and are separated by one meter. Since the radiation pattern of the antennas we use is planar, we place two transmitters at different heights of approximately $0.3 \mathrm{~m}$ and $0.46 \mathrm{~m}$. We note that the need for two transmitters is an artifact of the antennas we used and is not a fundamental requirement for Wision. The two transmitters send at different times and the receiver generates two different images using their transmissions. We then combine the images using the normalization technique described in $\$ 4.3$ to account for differences between the transmitters.

Fig.11 a) shows the results of our experiments. The plots show that the image created for the T-shaped metallic object approximates the underlying shape. The image created is blurrier than the actual object due to the Airy disk effect described in $\$ 5$ To see the effect of the antenna array size, in Fig. 11 b), we plot the image generated using a $4 \times 4$ antenna array placed at the same position as before. As expected, the images are blurrier than those with an $8 \times 8$ antenna array. This is because a $4 \times 4$ antenna array has a smaller aperture size and hence has a lower angular resolution. We note that the length and height of the object in the resulting image are within 5 and $8 \mathrm{~cm}$ of the actual object using the $8 \times 8$ antenna array and 18 and $40 \mathrm{~cm}$ using a $4 \times 4$ antenna array. This is in comparison to the 15 meter resolution using FMCW radar systems with a comparable bandwidth.

Observed Limitations: First, our two-dimensional antenna array experiments were performed by manually moving a single antenna over a linear actuator at different heights simulating an antenna array. This entire process roughly takes ten minutes per image. During this process, we assumed that the environment remains fairly constant. Further, since the final image computes the intensity value averaged across all the antenna locations, the small changes (e.g., people walking by) that almost always happened during this time period got averaged out from the image. We note that this is not a fundamental limitation of our approach and can be easily addressed by using actual antenna arrays. Second, as described earlier, the object in this experiment was a homogeneous metallic desktop. Objects that are made up of combination of materials (e.g., metal and plastic) have different 


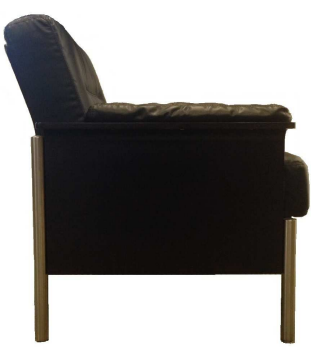

(a) Actual couch

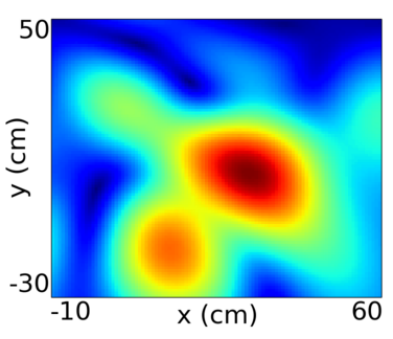

(b) Non-Line-of-sight
Figure 12-Non-line-of-sight imaging of a couch. The figure shows the imaged couch separated from the transmitter and the receiver by a $4 \mathrm{~cm}$ barrier. The figure shows that the different parts of the couch are distinctly clear even when an obstacle separates it from the transmitter and the receiver.

reflective properties; thus, parts of the object would appear with a higher intensity than the rest. Extracting the shape of such objects would require nulling the higher intensity material; understanding the limits of such an approach, however, is not in the scope of this paper.

\subsection{Imaging in NLOS Scenarios}

Next we evaluate Wision in non-line-of-sight (NLOS) scenarios. Specifically, we show the feasibility of imaging a common object (i.e., a couch) that is separated from the transmitter and the receiver by an obstacle (a polished wooden board barrier).

We use our two-dimensional antenna array to perform NLOS imaging of the couch shown in Fig. 12(a). We run experiments with the transmitter and the receiver on one side of a barrier $4 \mathrm{~cm}$ thick and the couch on the other side of the barrier. The couch is placed $0.4 \mathrm{~m}$ from the barrier and the transmitter and receiver are approximately $0.8 \mathrm{~m}$ on the other side of the wall. The results for other distance values are similar to that shown in this section. Since the transmitter and receiver are on the same side of the wall, the receiver sees two strong signals: one directly from the transmitter and another reflected from the wall. Since walls/barrier are reasonably smooth surfaces, they reflect specularly (i.e., mirrorlike). Thus, the receiver sees a signal that is reflected from the mid-point on the wall between the transmitter and the receiver. The receiver uses the nulling technique in $\$ 4.3$ to eliminate both the direct transmitter signals and the specular reflections from the barrier.

Fig. 12 plots the images for our experiments. The figures show that, while the reflections are weaker, they capture the general shape of the couch: we see reflections corresponding to the left leg, the mid-section, the head-rest and faint reflections from the right leg. These results show promise for imaging objects in non-line-of-sight scenarios that is not feasible with traditional cameras.

Observed Limitations: Our NLOS experiments use a relatively thin polished wooden barrier. As the barrier becomes thicker and more reflective in nature (e.g., concrete, metal), a smaller fraction of the signal reaches the object on the other side of the barrier making the resulting images fainter. By improving our interference nulling capabilities to be greater than our current $28-30 \mathrm{~dB}$ cancellation, one can reduce the

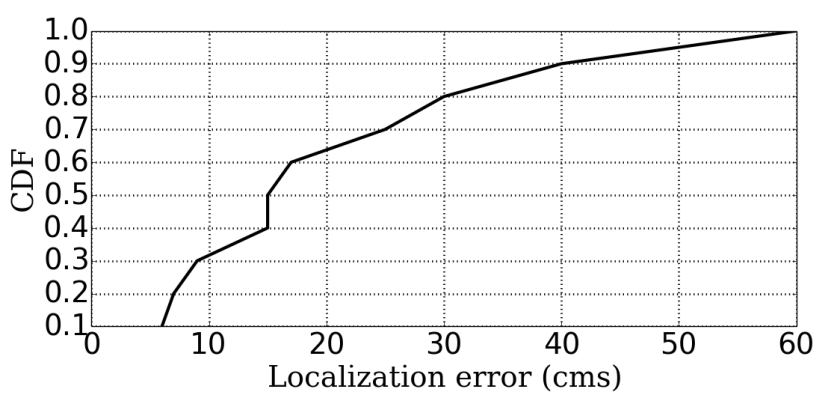

Figure 13-Performance of Tag-Free Object Localization. The figure plots the CDF of the localization error for a desktop in a 35 sq. meter office room. The plot shows that the median localization error is $15 \mathrm{~cm}$.

strong reflections from the barrier. This would result in making the intensities in the resulting images stronger. We also note that we place the object close to the barrier while capturing the imaging. This is mainly because our OFDM implementation on USRPs does not work well at higher power levels due to hardware non-linearities. Thus, we had to significantly reduce the transmission power from the USRP. With higher transmit power and better nulling capabilities we believe that the above limitations can be relaxed to some extent.

\section{Proof-of-Concept Applications}

Wision enables imaging of objects and humans using narrowband wireless signals (e.g., Wi-Fi). Given the ubiquity of Wi-Fi signals, we believe that this opens up multiple pervasive sensing applications that are traditionally considered difficult. In this section, we demonstrate proof-of-concepts for three applications that are enabled by Wision: (1) Object localization without the need to tag them with devices, (2) Localizing humans even when they are static, and (3) Through-the-fabric object detection. The goal of this exercise is to provide a glimpse into the possibilities opened by this technique; we consider fully exploring the potential uses and generalizing our results to various scenarios to be out of the scope of this paper.

\subsection{Localizing Objects without Tagging them}

To demonstrate the feasibility of object localization, we run experiments with a desktop of dimensions $44 \times 44 \times 18$ $\mathrm{cm}^{3}$. To perform localization, we first perform imaging using our two-dimensional antenna array and search for the desktop. Specifically, we look for a vertical band of highintensity reflection coefficients that match the dimensions of the desktop within an accuracy of 14 centimeters. We determine the location of our object by using a second receiver placed 2 meters from the first. We then estimate the intersection region between the images of the desktop created by the two receivers. Since our goal is to demonstrate the feasibility of object localization, extending our object recognition algorithm to general object shapes requires integrating Wision with computer vision techniques for object recognition. We also note that since Wision provides depth information, it can help improve the traditional object recognition accuracies by reducing the search space. Exploring this, however, would be the focus of future work.

We place our object in an office room of dimensions 7 by 


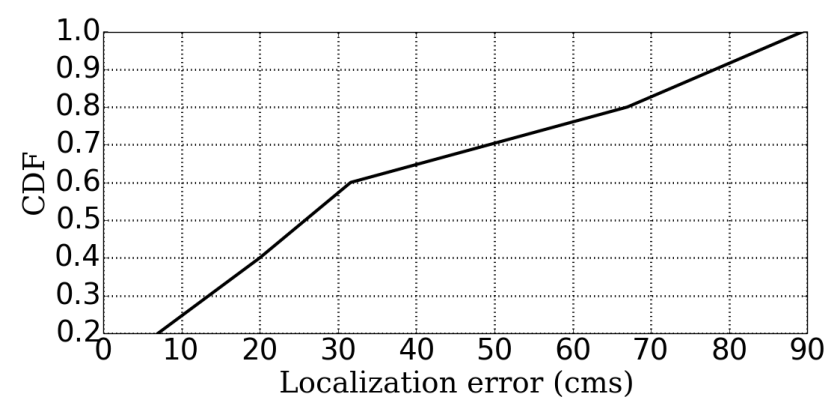

Figure 14-Performance of Static Human Localization. The figure plots the $\mathrm{CDF}$ of the localization error for a static human subject in a 35 sq. meter office room. The plot shows that the median localization error is around $26 \mathrm{~cm}$, which demonstrates the feasibility of tracking static humans without the need for carrying RF devices. During the experiments, multiple other human occupants moved around in the environment.

5 meters at 10 different locations. The room has other typical furniture including a couch, tables, and chairs. To focus on our object of interest, we use the beamforming and nulling algorithms described in $\$ 4.4$ and $\$ 4.3$. At each location, we compute the localization error by measuring the difference between the actual and estimated object locations. To eliminate the effect of other mobility in the environment, we average the intensity values output by our algorithm across time. We note that our environment does not have other objects with similar dimensions.

Fig. 13 plots a CDF of the localization error computed across different locations. The plot shows that the median error is about $15 \mathrm{~cm}$. We also see a variance in the localization error that is due to the orientation issue. Specifically the orientation of the object with respect to the receiver differs with the object locations, resulting in lower reflection intensities at some of the tested locations. This results in a maximum localization error of about $59 \mathrm{~cm}$.

\subsection{Static Human Localization}

Next to demonstrate the feasibility of static human localization, we run experiments with a male human subject with height $174 \mathrm{~cm}$ and weight about $160 \mathrm{lbs}$ We again use our antenna array and search for a block of intensities that has the corresponding height and width. We again run our experiment in the same office room as above with the subject at 10 different locations. As above, we leverage our beamforming and nulling algorithms to focus on the human in the presence of other objects in the environment. We note that our tested environment does not have furniture with similar dimensions to that of the standing human subject. Further, some of the tested locations were with the human standing next to the walls. Also, in $\$ 10$ we describe ideas for distinguishing humans and objects of similar dimensions.

Fig. 14 plots a CDF of the localization error computed across 10 different locations. The plot shows that the me-

\footnotetext{
${ }^{2}$ We focus on static human localization instead of mobile humans for two main reasons. First, detecting static humans is much more difficult since mobility create significant changes in the wireless signals that can help mobile human localization. Second, the ability to localize static humans at every time instance would also translate to localizing mobile humans by interpolating the images over time.
}
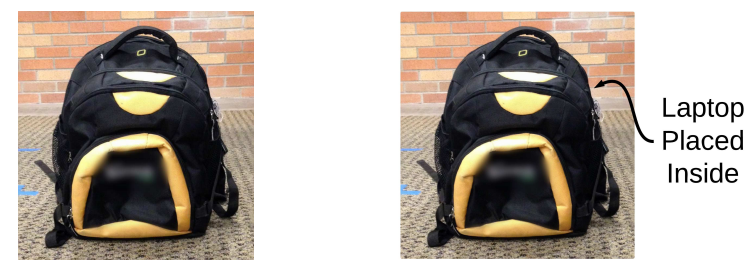

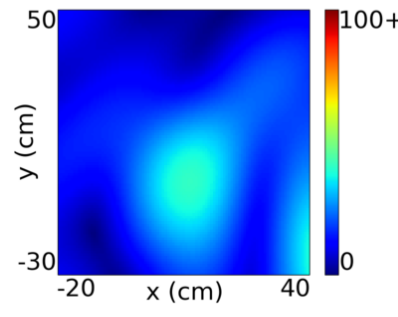

(a) Backpack without laptop

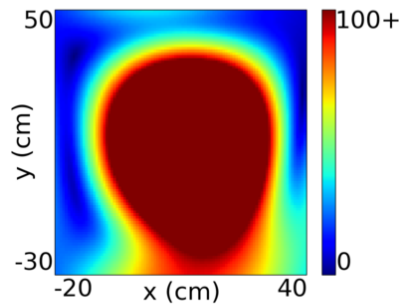

(b) Backpack with laptop
Figure 15-Imaging a backpack with and without a laptop. The figures show a significant intensity difference between the two scenarios, which demonstrates the ability to detect metallic objects in backpacks.
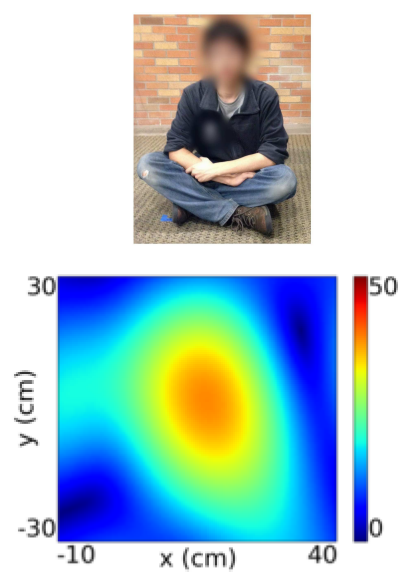

(a) Without the cellphone
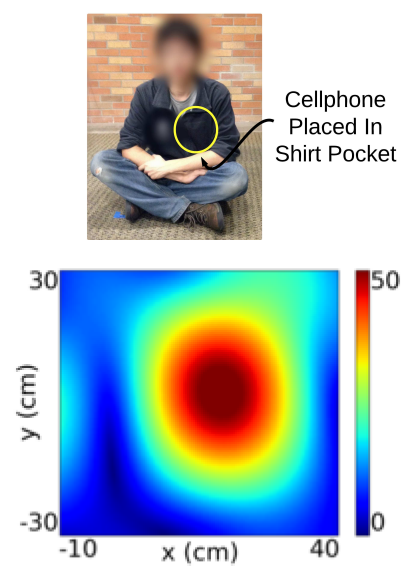

(b) Cellphone in hoodie pocket
Figure 16 - Imaging a cellphone in a hoodie pocket. The figures show an intensity difference between the two scenarios, which demonstrates the ability to detect metallic objects in the hoodie pocket. In addition, (a) demonstrates the ability to detect the faint outlines of humans.

dian localization error is around $26 \mathrm{~cm}$, which demonstrates the feasibility of tracking static humans without the need for carrying RF devices. We note that the localization errors are slightly higher than in the above object localization scenario since metallic objects have a higher reflection coefficient than the human body. We note that during our experiments, multiple human occupants moved around in the environment. This however did not affect our results, since Wision measures the intensity values at different azimuthal and elevation angles and averages them across time. This reduces the effect of mobile users in the environment. In principle, we can combine Wision with previous approaches on Wi-Fi motion detection [38, 19] to track both static and mobile humans in the environment.

\subsection{Imaging in through-the-fabric scenarios}

Finally, one of the benefits of using wireless signals for imaging is that, unlike light, it can "see through" clothes and 


\begin{tabular}{|l|cccc|}
\hline Material & Metal & Wood & Plastic & Foam \\
\hline Relative Intensity & 235 & 93 & 27 & 14 \\
\hline
\end{tabular}

Table 1-Relative reflection intensity of different materials.

fabric. We evaluate the feasibility of two such scenarios: (1) detecting a laptop that is within a backpack, and (2) imaging a phone in a hoodie pocket.

Detecting a laptop in a backpack. Laptops have a number of metallic components that have better reflective properties than the fabric of a backpack. So the presence of a laptop in the backpack significantly increases the reflective intensity in the image. We run experiments with a 13-inch MAC Book Pro placed inside a closed backpack. We use a two-dimensional antenna array as our receiver and a singleantenna transmitter. The transmitter uses a log periodic directional antenna pointing towards the backpack. We place the backpack in front of the receiver on the floor at a distance of one meter. We first image the empty backpack and then image the backpack with the laptop placed in it. The wireless connectivity on the laptop is turned OFF during the experiments. Fig. 15(a) and (b) show the two images. The figure shows a faint reflection when there is only a backpack. When there is a laptop in it, however, the reflection intensity significantly increases. This is expected because metallic objects have stronger reflection properties. The above experiment demonstrates the basic feasibility of detecting metallic devices within backpacks.

Detecting a Phone in the pocket. Next we evaluate the feasibility of detecting a phone in a hoodie pocket. To do this, we use an iPhone 5 that is placed in the hoodie pocket worn by a volunteer. The subject sits on the ground and has the phone in the hoodie pocket. As above, we run experiments with an $8 \times 8$ antenna array as our receiver. We use a single directional antenna as our transmitter. The subject is 1.5 meters away from the receiver. We run the experiments both with and without the phone in the hoodie. We turn OFF the Wi-Fi transmissions on the phone to ensure that the only signals we see from the phone are due to the reflected signals of the transmitter.

Fig. 16(a) and (b) shows the images with and without the phone in the hoodie. The figures show the following:

- In the absence of the phone, the images capture the faint reflections from the human body. Specifically, the faint signals we see in the image outline the sitting subject. This is noteworthy since posture information can be useful in a number of activity detection applications.

- With the phone, we see a bright reflective blob in the center; this higher reflective intensity is because the phone is a metallic object and hence reflects more than the human body. These results show the feasibility of detecting metallic objects hidden on the human body.

\section{Micro-Benchmarks}

Finally, we evaluate key properties that affect an object's interaction with $\mathrm{Wi}-\mathrm{Fi}$ - size, material, and orientation.

Effect of Size. We consider four metal objects of decreasing dimensions - a desktop with dimensions $44 \times 44 \times 18 \mathrm{~cm}^{3}$,

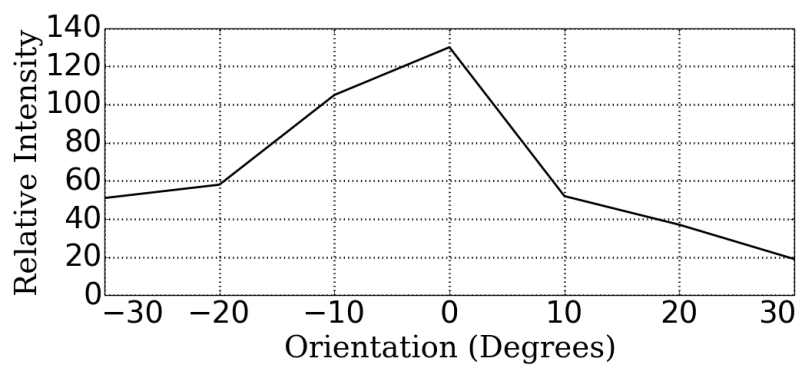

Figure 17-Effect of Object Orientation. The figure plots the average reflection intensities of a smooth metallic object as we vary its orientation. However, given the intensity values observed, Wision could still detect the object for all the orientations.

a laptop with dimensions $33 \times 23 \times 2 \mathrm{~cm}^{3}$, an empty water bottle with diameter $8 \mathrm{~cm}$ and height $25 \mathrm{~cm}$, and a hollow metal rod with diameter $2 \mathrm{~cm}$ and height $58 \mathrm{~cm}$. We create images for each of these objects using an $8 \times 1$ antenna array as our receiver and a single-antenna transmitter. Both our receiver and the transmitter use the WA5VJB log periodic antennas. We place our $8 \times 1$ array receiver and the singleantenna transmitter at coordinates $(0,0)$ and $(80,30)$ respectively. The tested objects are placed around one meter away at an angle of 60 degrees from the receiver. We perform the nulling operation in $\$ 4.3$ to remove the direct signals from the transmitter and also the walls.

Fig. 18 shows the intensities for the different tested objects. The figures show the intensity of the reflections arriving at the receiver from different angles. The plots show:

- The reflective intensity decreases with the size of the object: a bottle reflects less than the desktop and the laptop, and the signals from a thin metal rod are even weaker. This is expected since $2.4 \mathrm{GHz}$ transmissions have a wavelength of around $12 \mathrm{~cm}$ and hence tend to pass through objects with smaller dimensions.

- The width of the strong intensity regions in the plots becomes smaller with the size of the object. This shows that Wision can capture information about the object's width.

- Finally, we note that the hollow rod was not noticeable before performing the nulling operation at the transmitter (figure not shown due to space considerations). However, as we see in Fig.18(d), after we perform the nulling operation, the object becomes visible albeit with low intensity. Thus, using nulling, Wision can improve its imaging effectiveness with small objects.

Effect of Material. As described in $\$ 5$ the material of the objects affects both its reflective intensities as well as its reflective properties (i.e., diffuse versus specular reflective properties). To see this, we experiment with four common objects in indoor spaces - an empty metal cabinet $\left(76 \times 68 \times 46 \mathrm{~cm}^{3}\right)$, a wooden desk $\left(119 \times 41 \times 70 \mathrm{~cm}^{3}\right)$, leather cushions $\left(58 \times 63 \times 20 \mathrm{~cm}^{3}\right)$ and a ridged plastic board $\left(66 \times 41 \times 5 \mathrm{~cm}^{3}\right)$. As before, we image these objects using an $8 \times 1$ antenna array. Table. 1 1 shows the average reflective intensities at the receiver along the object's direction. The numbers show that the metallic object results in a higher intensity in comparison to the wooden object, which in turn 
is a better reflector than the plastic object and the cushions (foam). This matches our intuition that an object's material affects its reflectivity.

Effect of Orientation. Finally, the orientation of an object affects the intensity of the reflected signal, especially when the object acts like a mirror. So we pick the smooth metallic desktop and compute the average intensity observed in the direction of the object. We repeat the experiment for different object orientations. We again replicate the set up in the previous experiment with a directional antenna at the transmitter and an $8 \times 1$ antenna array as the receiver.

Fig. 17 plots the average intensity values as a function of the smooth metallic object's orientation. The figure shows that the reflective intensity significantly varies with the orientation of the object. This is expected because smooth surfaces have larger specular (mirror-like) reflections. The high intensity value in the figure corresponds to the orientation where the receiver lies along the direct reflected ray from the object. We note that while the intensity values change, we can still image the object for all the orientation values.

\section{Limitations and Discussions}

Finally, we summarize the limits of Wi-Fi imaging; some of these correspond to our specific implementation and others that are more fundamental.

Object Size and Material. Our evaluation demonstrates Wision's ability to image and localize either relatively large objects such as couches, desktops, and T-shaped objects or objects that have good reflective properties (e.g., metallic surfaces). Smaller objects of the same material have smaller cross-sections and reflect a smaller fraction of the incident wireless signals; hence making them harder to image. Further, as the size of the object becomes proportional to the wavelength of Wi-Fi signals (approx. $12 \mathrm{~cm}$ at $2.4 \mathrm{GHz}$ ), its interaction with the Wi-Fi signals reduces. This is a fundamental limitation of imaging with Wi-Fi transmissions. One could address this by going to higher frequencies (i.e., $5 \mathrm{GHz}$ $\mathrm{Wi}-\mathrm{Fi}$ ) that have a smaller wavelength (approx. $6 \mathrm{~cm}$ ). We however believe that Wision still represents a significant capability that will be useful in many areas ranging from inventory tracking (e.g., tracking equipment such as trolleys and carts without having to tag them with sensors) to smart homes (e.g., monitoring the status of large items like doors, windows, etc., without additional sensor deployment).

Imaging Resolution. As described in \$5, the imaging resolution with Wi-Fi signals depends on two main parameters: the signal wavelength and the antenna array length. For a given wavelength, one can increase the imaging resolution by increasing the length of the antenna array. We believe that the resolution we achieved is close to the optimal at $2.4 \mathrm{GHz}$ for the considered array lengths and is unlikely to increase without either increasing the length of the antenna array or using $5 \mathrm{GHz}$ Wi-Fi transmissions. We also note that the resolution mainly depends on the length of the antenna array and not on the number of antennas. Specifically, recent theoretical work has shown that one can achieve similar resolutions with lesser number of antennas as long as the length of the antenna array is kept constant [30].

Object Orientation. One of the key constraints we found with our prototype implementation is that Wi-Fi transmissions in the environment reach an object only if that object lies within the radiation pattern of the transmitter. Further, since smooth metallic surfaces act like mirrors, they could be oriented such that they are hidden from view for certain transmitter positions. To address these issues, one may either pick antennas with better radiation patterns or optimize the antenna position to maximize their reach. Another approach is to leverage transmissions from multiple $\mathrm{Wi}-\mathrm{Fi}$ devices in the environment, which are naturally likely to be at different positions. Further, one can leverage the mobility of wireless devices (e.g., smartphones) to create images by stitching together the intensities as the user moves around. Exploring these approaches, however is not in the scope of this paper.

\section{Conclusion}

We present Wision, a novel imaging system that uses narrowband wireless transmissions (e.g., Wi-Fi). Wision leverages multi-path propagation that results in wireless signals bouncing off of objects before arriving at the receiver. These reflections effectively light up the objects, which Wision uses to image them. We also show how to extract depth information where objects that are at different distances from the receiver can be identified. We implement a prototype of Wision and image objects such as leather couches and metallic shapes in both line-of-sight and non-line-of-sight scenarios. We also demonstrate proof-of-concept applications including localization of static humans and objects using Wision, without the need for tagging them with RF devices. Finally, we explore the limits of our Wi-Fi based imaging approach.

Acknowledgements: We would like to thank the anonymous SenSys reviewers for their helpful comments. This research is funded in part by the NSF award CNS-1420654, Google Faculty Award, and University of Washington.

\section{References}

[1] Hg2415g grid antenna. http://www.l-com.com/multimedia/ datasheets/DS_HG2415G-NF-5PK.PDF.

[2] wa5vjb directional antenna. http://www.wa5vjb.com/pcb-pdfs/ LP8565.pdf.

[3] Xirrus corporation. http://www.xirrus.com.

[4] F. Ahmad and M. Amin. Through-the-wall human motion indication using sparsity-driven change detection. In IEEE Transactions on Geoscience and Remote Sensing, 2013.

[5] F. Ahmad, M. Amin, and P. Setlur. Through-the-wall target localization using dual-frequency cs radars. In Proc. SPIE 6201, C3I Technologies for Homeland Security and Homeland Defense V, 62010H, 2006.

[6] G. Airy. On the diffraction of an object-glass with circular aperture. In Transactions of the Cambridge Philosophical Society, 1835.

[7] I. Amundson, J. Sallai, X. Koutsoukos, and A. Ledeczi. Rf angle of arrival-based node localization. In International Journal of Sensor Networks, 2011

[8] P. Bahl and V. N. Padmanabhan. Radar: An in-building rf-based user location and tracking system. In INFOCOM, 2000.

[9] P. Beckmann and A. Spizzichino. The scattering of electromagnetic waves from rough surfaces. Artech house, 1987.

[10] D. Bharadia, K. Joshi, and S. Katti. Full duplex backscatter. In Hotnets, 2013.

[11] D. Bharadia, E. McMilin, and S. Katti. Full duplex radios. In Sigcomm, 2013.

[12] W. Carrara, R. Goodman, and R. Majewski. Spotlight Synthetic Aperture Radar: Signal Processing Algorithms. Artech House, 1995. 


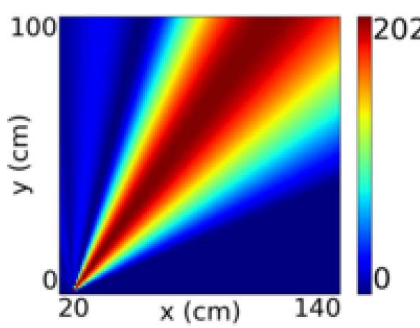

(a) Desktop

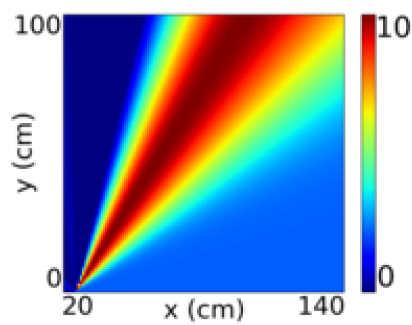

(b) Laptop
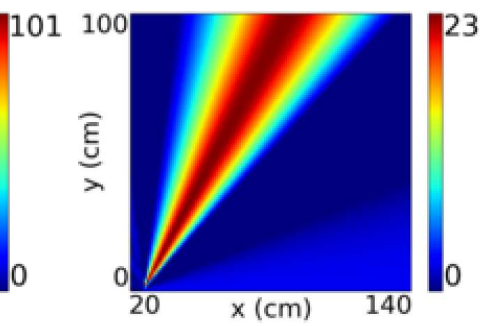

(c) Bottle

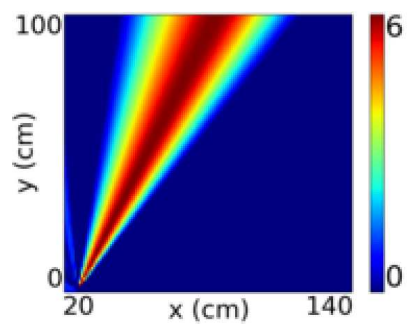

(d) $\operatorname{Rod}$

Figure 18-Effect of size. The plots show the intensity maps captured using a one-dimensional antenna array as the receiver. The plots show that the intensity of the multi-path reflections decrease with the size of the object. Further, the width of the reflections is proportional to the width of the object. Wision however could extract the intensity values for all the tested objects using its nulling algorithm.

[13] G. Charvat, L. Kempel, E. Rothwell, C. Coleman, and E. Mokole. A Through-dielectric Radar Imaging System. In Trans. Antennas and Propagation, 2010.

[14] K. Chetty, G. Smith, and K. Woodbridge. Through-the-wall Sensing of Personnel Using Passive Bistatic WiFi Radar at Standoff Distances. In Trans. Geoscience and Remote Sensing, 2012.

[15] K. Chintalapudi, A. Iyer, and V. Padmanaban. Indoor Localization without the Pain. In NSDI, 2011.

[16] J. R. Costa, E. B. Lima, C. R. Medeiros, T. Radil, R. C. Martins, P. M. Ramos, and C. A. Fernandes. Development of an Indoor Wireless Personal Area Network based on Mechanically steered millimeter-wave lens antenna. In I2MTC, 2010.

[17] O. K. Ersoy. Diffraction, Fourier Optics and Imaging. John Wiley Sons, 2006.

[18] F. FAdib, Z. adelec, D. Katabi, and R. Miller. 3d localization via human body reflections. In NSDI, 2014

[19] F. FAdib and D. Katabi. Seeing Through Walls Using WiFi! In SIGCOMM, 2013

[20] J. P. Fitch. Synthetic Aperture Radar. 1988.

[21] J. Friedman, Z. Charbiwala, T. Schmid, Y. cho, and M. Srivastava. Angle-of-arrival assisted radio interferometry target localization. In MILCOM, 2008.

[22] J. Gjengset, J. Xiong, G. McPhilips, and K. Jamieson. Enabling Phased Array Signal Processing on Commodity WiFi access points. In Mobicom, 2014.

[23] A. Gonzalez-Ruiz and Y. Mostofi. Cooperative robotic structure mapping using wireless measurements, a comparison of random and coordinated sampling patterns. In IEEE Sensors Journal, 2013.

[24] A. Gonzelez-Ruiz, A. Ghaffarkhah, and Y. Mostofi. An integrated framework for obstacle mapping with see-through capabilities using laser and wireless channel measurements. In IEEE Sensors, 2014.

[25] D. Harperin, W. Hu, A. Sheth, and D. Wetherall. Gathering 802.11n traces with channel state information. In CCR, 2011.

[26] E. Hecht. Optics, 2nd Edition. 1987.

[27] J. Hunt, T. Driscoll, A. Mrozack, G. Lipworth, M. Reynolds, D. Brady, and D. Smith". Metamaterial apertures for computational imaging. In Science, 2013

[28] B. Kellogg, V. Talla, and S. Gollakota. Bringing gesture recognition to all devices. In NSDI, 2014.

[29] D. Y. Kim and O. Kenneth. 280ghz and 860ghz image sensors using schottky-barrier diodes in $0.13 \mathrm{~m}$ digital cmos. In International SolidState Circuits Conference, 2012.

[30] J. Krieger, Y. Kochman, and G. Wornell. Multi-Coset, Sparse Imaging Arrays. In IEEE transactions on Antennas and Propogation, 2014.

[31] C.-P. Lai and R. Narayanan. Through-wall imaging and characterization of human activity using ultrawideband (uwb) random noise radar. In C3I Technologies for Homeland Security and Homeland Defense, 2005.

[32] N. Levanon and E. Mozeson. Radar Signals. John Wiley Sons, 2004.

[33] K. Lin, S. Gollakota, and D. Katabi. Random Access Heterogeneous MIMO Networks. In SIGCOMM, 2011.

[34] V. Lubecke, O. Boric-Lubecke, H. Madsen, and A. Fathy. Throughthe-wall radar life detection and monitorin. In IEEE/MTT-S, 2007.
[35] P. Maechler, N. Felber, and H. Kaeslin. Compressive sensing for wifibased passive bistatic radar. In EUSIPCO, 2012.

[36] Y. Mostofi. Cooperative wireless-based obstacle/object mapping and see-through capabilities in robotic networks. In IEEE Transactions on Mobile Computing, 2013.

[37] N. Patwari, L. Brewer, Q. Tate, O. Kaltiokallio, and M. Bocca. Breathfinding: A Wireless Network That Monitors and Locates Breathing in a Home. In IEEE Journal Signal Processing, 2014.

[38] Q. Pu, S. Gupta, S. Gollakota, and S. Patel. Whole-Home Gesture Recognition Using Wireless Signals. In MOBICOM, 2013.

[39] T. Ralston, G. Charvat, and J. Peabody. Real-time through-wall imaging using an ultrawideband MIMO phased array radar system. In Array, 2010.

[40] S. Ram and H. Ling. Through-wall tracking of human movers using joint doppler and array processing. In Geoscience and Remote Sensing, 2008.

[41] C. Rodenbeck and K. Chang. Automated pattern measurement for circularly-polarized antennas using the phase-amplitude method. In Microwave Journal, 2004.

[42] A. Saeed, A. E. Kosba, and M. Youssef. Ichnaea: A low-overhead robust wlan device-ftree passive localization system. In IEEE Journal of selected topics in signal processing, 2014.

[43] R. O. Schmidt. Multiple Emitter Location and Signal Parameter Estimation. In IEEE Trans. on Antennas and Propagation, AP-34(3):276280,Mar. 1986.

[44] S. Sen, B. Radunovic, R. R. Choudhury, and T. Minka. Spot localization using PHY layer information. In Mobisys, 2012.

[45] C. Shepard, H. Yu, N. Anand, E. Li, T. Marzetta, R. Yang, and L. Zhong. Argos: practical many-antenna base stations. In Mobicom, 2012.

[46] S. Sigg, S. Shi, and Y. Ji. Rf-based device-free recognition of simultaneously conducted activities. In Ubicomp, 2013.

[47] M. Skolnik. Radar Handbook. McGraw-Hill, 1988.

[48] H. Wang, S. Sen, A. Elgohary, M. Youssef, and R. R. Choudhury. No need to war-drive: unsupervised indoor localization. In MobiSys, 2012.

[49] Y. Wang, M. Kuhn, and A. Fathy. Advanced system level simulation of uwb three-dimensional through-wall imaging radar for performance limitation prediction. In MTT, 2010.

[50] J. Wilson and N. Patwari. Through-Wall Motion Tracking Using Variance-Based Radio Tomography Networks. In ARXIV, 2009.

[51] J. Xiong and K. Jamieson. ArrayTrack: A Fine-Grained Indoor Location System. In NSDI, 2013.

[52] Q. Yang, X. Li, H. Yao, J. Fang, K. Tan, W. Hu, J. Zhang, and Y. Zhang. BigStation: Enabling Scalable Real-time Signal Processing in Large MU-MIMO Systems. In SIGCOMM, 2013.

[53] M. Youssef and A. Agrawala. The horus wlan location determination system. In MobiSys, 2005.

[54] Y. Zhao and N. Patwari. Robust Estimators for Variance-Based Device-Free Localization and Tracking. In ARXIV, 2011.

[55] F. Zhu, S. Gao, A. Ho, W. Brown, J. Li, and J. Xu. Low-profile directional ultra-wideband antenna for see-through-wall imaging applications. In Electromagnetics Research, 2011. 